\title{
Post-fracture pharmacotherapy for women with osteoporotic fracture: analysis of a managed care population in the USA
}

\author{
A. Wilk • S. Sajjan • A. Modi • C.-P. S. Fan • P. Mavros
}

Received: 1 April 2014 / Accepted: 25 July 2014 / Published online: 12 August 2014

(C) The Author(s) 2014. This article is published with open access at Springerlink.com

\begin{abstract}
Summary Pharmacologic therapy is recommended to reduce future fracture risk. We examined osteoporosis medications dispensed to older women after first fracture. Only $23 \%$ received therapy during the first year post-fracture. Prior osteoporosis therapy, a prior osteoporosis diagnosis, and older age were good predictors of post-fracture osteoporosis therapy.

Introduction Pharmacologic therapy is recommended after osteoporotic fracture to reduce future fracture risk. The objective of this retrospective study was to examine osteoporosis therapy dispensed to women post-fracture.

Methods We identified women $\geq 50$ years old in a large administrative claims database from 2003 to mid-2012 who were continuously enrolled 2 years before (baseline) and 1 year after first osteoporotic fracture. Exclusions were Paget's disease or malignant neoplasm. Pre- and post-fracture osteoporosis therapies (oral and parenteral) were assessed overall and by fracture site.

Results A total of 47,171 women of mean (SD) age of 63 (10) years were eligible; fractures included $8 \%$ hip, $17 \%$ vertebral, $73 \%$ non-hip/non-vertebral, and $3 \%$ multiple fracture sites. Only $18 \%$ received osteoporosis therapy within 90 days
\end{abstract}

Electronic supplementary material The online version of this article (doi:10.1007/s00198-014-2827-x) contains supplementary material, which is available to authorized users.

A. Wilk

Department of Biostatistics, Virginia Commonwealth University, Richmond, VA, USA

S. Sajjan $\cdot$ A. Modi $(\bowtie) \cdot$ P. Mavros

Center for Observational and Real-World Evidence, Merck \& Co., Inc., One Merck Drive, PO Box 100, WS2E-76, Whitehouse Station, NJ 08889-0100, USA

e-mail: ankita.modi@merck.com

C.-P. S. Fan

AsclepiusJT LLC, New York, NY, USA and $23 \%$ within 1 year post-fracture. Overall, $19 \%$ of women had a prior osteoporosis diagnosis; $20 \%$ had received osteoporosis therapy during baseline. Of 37,649 (80\%) women without baseline therapy, only $9 \%$ initiated pharmacologic therapy within 1 year. The adjusted odds ratio (OR) of therapy within 1 year post-fracture was significantly greater for women who had received baseline osteoporosis therapy (versus none) and who had vertebral (OR 12.7, $95 \%$ confidence interval (CI) 11.2-14.5), hip (15.2, 12.5-18.7), or non-hip/ non-vertebral fracture $(34.4,31.7-37.3)$. Other significant predictors included pre-fracture osteoporosis diagnosis (1.6, 1.4-1.7) and older age (OR range, 1.3-1.7). Treatment adherence was significantly better among women with baseline osteoporosis diagnosis.

Conclusions The substantial post-fracture treatment gap represents an important unmet need for women with osteoporotic fractures. Fracture liaison or adherence programs could lead to improved post-fracture treatment rates.

Keywords Hip fracture $\cdot$ Osteoporosis $\cdot$ Post-fracture therapy $\cdot$ Under-treatment $\cdot$ Vertebral fracture

\section{Introduction}

The burden on patients and health care systems associated with osteoporotic fractures is substantial and likely to remain so for the foreseeable future due to the aging worldwide population [1-3]. In the USA, the prevalence of osteoporosis at either the lumbar spine or femoral neck among women 50 years of age and older has been estimated at $17 \%$, affecting seven million women; one in two women is projected to experience an osteoporotic fracture during her lifetime [4, 5].

The occurrence of osteoporotic fractures is well established as an important risk factor for future fractures [6-11]. In the USA, the National Osteoporosis Foundation guidelines 
recommend consideration of pharmacotherapy after hip or vertebral fracture in post-menopausal women and men 50 years of age and older [5]. Several pharmacologic therapies have proven efficacy for reducing risk of osteoporotic fracture $[12,13]$; however, women and men at risk of fracture often do not receive preventive treatment $[14,15]$. In many countries, the post-fracture care gap is a problem, as patients with hip or other fractures are often not prescribed osteoporosis therapy [15-20]. Moreover, poor adherence and lack of persistence with therapy are common even among patients who are prescribed with osteoporosis therapy $[21,22]$.

The objective of this retrospective study was to examine osteoporosis therapy dispensed to women post-fracture. We sought to describe patient demographic and clinical characteristics and osteoporosis treatment status, including medication use and adherence, prior to first osteoporotic fracture and within 90 days and 1 year post-fracture for women in a large managed care population in the USA. In addition, we aimed to assess predictors of post-fracture medication use within 1 year post-fracture.

\section{Methods}

Data source and study population

We used de-identified, longitudinal patient information contained in the i3 Invision Datamart database (now known as the Clinformatics ${ }^{\mathrm{TM}}$ Data Mart [23]). This administrative claims dataset contains medical and pharmacy claims and laboratory results for a large geographically diverse patient population in the USA including approximately three million patients with osteoporosis. Available data included patient enrollment and administrative claims data for outpatient visits, hospitalizations, laboratory tests, and pharmacy claims. Disease diagnoses and comorbidities were identified in the dataset using the International Classification of Diseases, Ninth Revision, Clinical Modification (ICD-9-CM) diagnosis codes [24]. Medications were identified using the National Drug Code Directory (NDC) codes in the pharmacy claims [25] and J-codes for intravenous drugs in the medical claims.

We studied women 50 years of age and older who experienced their first osteoporotic fracture between January 1, 2005 and June 30, 2011. To be eligible, women had to be continuously enrolled in the database for at least 2 years prior to (baseline period) and at least 1 year post-fracture; the date of first recorded (index) fracture was defined as the index date. Thus, the entire study window was from January 1, 2003 to June 30, 2012.

Osteoporotic fractures were identified by ICD-9-CM diagnosis codes $[24,26]$ and categorized by site into four groups: vertebral fracture only, hip fracture only, non-hip/non-vertebral, and fractures at multiple sites.
We excluded patients with a record of fracture before the index date, a diagnosis of Paget's disease (osteitis deformans; ICD-9-CM code 731.0) at any time in the database, or a diagnosis of malignant neoplasm (ICD-9-CM codes 140.xx to 208.xx, 230.xx to 239.xx, or 172.xx) during their 3-year study period.

\section{Study baseline characteristics}

We assessed patients' age at the index date, insurance type, the presence or absence of 28 comorbidities (listed in the Online Resource Table S1), and the Charlson comorbidity index score to quantify overall comorbidity [27]. In addition, for the baseline period, we assessed the presence of bone mineral density tests (CPT codes 76070-76071, 76075-76076, 76078, 76977, and 77078-77083), the history of falls resulting in medical services (ICD-9-CM codes E880-E888) and the use of gastroprotective agents (proton pump inhibitors, histamine H2-receptor antagonists, and cytoprotectant agents), corticosteroids, estrogen, and non-steroidal anti-inflammatory drugs (NSAIDs).

We examined whether patients had received osteoporosis therapy during the baseline period, within 90 days post-fracture, and within 1 year post-fracture. Patients who had received baseline therapy were classified into two groups according to whether the days' supply of medication overlapped the index date (currently treated) or did not overlap the index date (not currently treated). All forms of osteoporosis therapy, oral and parenteral, were considered in the study and included the bisphosphonates (alendronate, risedronate, ibandronate, zoledronic acid), calcitonin, denosumab, raloxifene, and teriparatide.

Dispensed prescriptions for all types of osteoporosis therapy were used to calculate medication possession ratio (MPR), namely, the proportion of days covered by dispensed medications. For the post-fracture period, the MPR was calculated as total days' supply/365. If the total days' supply exceeded 365 days, it was truncated to 365 days, yielding a maximum MPR of 1.0. The MPR for pre-index date therapy during the baseline period was calculated as the total days' supply/730. We defined adherence to therapy as an $\mathrm{MPR} \geq 0.80$.

\section{Statistical analysis}

Baseline patient characteristics (including demographic and clinical parameters) were summarized and described overall and by index fracture group (vertebral, hip, non-hip/non-vertebral, and multiple fractures). Continuous variables were summarized by means and standard deviations (SDs), overall and by fracture group; comparisons were made using F-tests or Kruskal-Wallis tests where appropriate. Categorical variables were summarized by the number and percentage of 
patients, overall and by fracture group; comparisons were made using $\chi^{2}$ tests. A statistical significance level of $5 \%$ was used for all aforementioned analyses.

Multivariable logistic regression was used to identify the association between 1-year treatment status (treated vs. not treated within 1 year post-fracture) and patients' baseline treatment status (not treated vs. previously/currently treated) among index fracture groups, adjusted for baseline patient characteristics. Interactions between the baseline treatment status and index fracture group were considered to estimate differential effects on 1-year treatment status among different treatment-fracture combinations. Using stepwise (forward and backward) variable selection with a tuning significance of $20 \%$, potentially important baseline patient characteristics were selected from a list of candidate covariates possibly associated with post-fracture treatment status. The covariates included age groups, health plan types, Charlson comorbidity index score, use of concomitant medications, and comorbid conditions, as listed in Online Resource Table S1. Only $13,380(28.4 \%)$ patients had a bone mineral density assessment, and the results were not available from the database; thus, bone mineral density was not used as an inclusion criterion or included in the model. The final model included the key predictors (baseline treatment status, index fracture

Table 1 Baseline demographic and clinical characteristics of all women and by index fracture type

\begin{tabular}{|c|c|c|c|c|c|}
\hline \multirow[b]{2}{*}{ Characteristic } & \multirow[b]{2}{*}{$\begin{array}{l}\text { All women } \\
(n=47,171)\end{array}$} & \multicolumn{4}{|l|}{ Fracture group } \\
\hline & & $\begin{array}{l}\text { Vertebral } \\
(n=7,834)\end{array}$ & $\operatorname{Hip}(n=3,599)$ & $\begin{array}{l}\text { Non-hip/non-vertebral } \\
(n=34,396)\end{array}$ & $\begin{array}{l}\text { Multiple } \\
(n=1,342)\end{array}$ \\
\hline Age at index date, mean (SD) & $63.4(9.8)$ & $66.8(10.2)$ & $71.3(9.6)$ & $61.6(9.0)$ & $67.8(10.4)$ \\
\hline $50-59$ years, $n(\%)$ & $20,931(44.4)$ & $2,469(31.5)$ & $610(16.9)$ & $17,451(50.7)$ & $401(29.9)$ \\
\hline $60-69$ years, $n(\%)$ & $12,446(26.4)$ & $1,901(24.3)$ & $657(18.3)$ & $9,612(27.9)$ & $276(20.6)$ \\
\hline 70-79 years, $n(\%)$ & $10,515(22.3)$ & $2,563(32.7)$ & $1,651(45.9)$ & $5,827(16.9)$ & $474(35.3)$ \\
\hline$\geq 80$ years, $n(\%)$ & $3,279(7.0)$ & $901(11.5)$ & $681(18.9)$ & $1,506(4.4)$ & $191(14.2)$ \\
\hline \multicolumn{6}{|l|}{ Insurance type, $n(\%)$} \\
\hline $\mathrm{HMO}$ & $9,411(20.0)$ & $1,739(22.2)$ & $926(25.7)$ & $6,442(18.7)$ & $304(22.7)$ \\
\hline POS & $21,611(45.8)$ & $2,952(37.7)$ & $897(24.9)$ & $17,311(50.3)$ & $451(33.6)$ \\
\hline PPO & $4,292(9.1)$ & $787(10.0)$ & $429(11.9)$ & $2,930(8.5)$ & $146(10.9)$ \\
\hline Other & $11,857(25.1)$ & $2,356(30.1)$ & $1,347(37.4)$ & $7,713(22.4)$ & $441(32.9)$ \\
\hline Prior osteoporosis diagnosis, $n(\%)$ & $9,168(19.4)$ & $2,230(28.5)$ & $745(20.7)$ & $5,877(17.1)$ & $316(23.5)$ \\
\hline BMD assessment performed ${ }^{\mathrm{a}}, n(\%)$ & $13,380(28.4)$ & $2,346(29.9)$ & $731(20.3)$ & $9,976(29.0)$ & $327(24.2)$ \\
\hline History of falls of any type resulting in medical services, $n(\%)$ & $3,470(7.4)$ & $587(7.5)$ & $313(8.7)$ & $2,470(7.2)$ & $100(7.5)$ \\
\hline Baseline osteoporosis medication, $n(\%)$ & $9,522(20.2)$ & $2,171(27.7)$ & $727(20.2)$ & $6,291(18.3)$ & $333(24.8)$ \\
\hline Currently treated ${ }^{\mathrm{b}}, n(\%)$ & $4,936(10.5)$ & $1,181(15.1)$ & $338(9.4)$ & $3,243(9.4)$ & $174(13.0)$ \\
\hline CCI score, mean (SD) & $1.0(1.5)$ & $1.3(1.7)$ & $1.5(1.8)$ & $0.9(1.4)$ & $1.2(1.7)$ \\
\hline \multicolumn{6}{|l|}{ Key comorbid conditions, $n(\%)$} \\
\hline Osteopenia & $8,803(18.7)$ & $1,600(20.4)$ & $506(14.1)$ & $6,471(18.8)$ & $226(16.8)$ \\
\hline Arthritis & $24,992(53.0)$ & $4,808(61.4)$ & $2,151(59.8)$ & $17,293(50.3)$ & $740(55.1)$ \\
\hline Myocardial infarction & $1,242(2.6)$ & $319(4.1)$ & $168(4.7)$ & $710(2.1)$ & $45(3.4)$ \\
\hline Cerebrovascular disease & $5,113(10.8)$ & $1,162(14.8)$ & $731(20.3)$ & $3,017(8.8)$ & $203(15.1)$ \\
\hline Chronic pulmonary disease & $10,118(21.4)$ & $2,124(27.1)$ & $857(23.8)$ & $6,853(19.9)$ & $284(21.2)$ \\
\hline Hypothyroidism & $9,870(20.9)$ & $1,874(23.9)$ & $747(20.8)$ & $6,972(20.3)$ & $277(20.6)$ \\
\hline Diabetes & $8,353(17.7)$ & $1,466(18.7)$ & $757(21.0)$ & $5,893(17.1)$ & $237(17.7)$ \\
\hline Obesity & $3,463(7.3)$ & $554(7.1)$ & $177(4.9)$ & $2,664(7.7)$ & $68(5.1)$ \\
\hline \multicolumn{6}{|l|}{ Concomitant medication use, $n(\%)$} \\
\hline Gastroprotective agent & $11,509(24.4)$ & $2,291(29.2)$ & $988(27.5)$ & $7,911(23.0)$ & $319(23.8)$ \\
\hline NSAID & $17,384(36.9)$ & $3,240(41.4)$ & $1,270(35.3)$ & $12,438(36.2)$ & $436(32.5)$ \\
\hline Glucocorticoid & $12,896(27.3)$ & $2,581(32.9)$ & 839 (23.3) & $9,152(26.6)$ & $324(24.1)$ \\
\hline Estrogen & $8,479(18.0)$ & $1,467(18.7)$ & $422(11.7)$ & $6,394(18.6)$ & $196(14.6)$ \\
\hline
\end{tabular}

$B M D$ bone mineral density, $C C I$ Charlson comorbidity index, $H M O$ health maintenance organization, NSAID non-steroidal anti-inflammatory drug ${ }^{\text {a }} \mathrm{BMD}$ results were not available from the database

${ }^{\mathrm{b}}$ Receiving prescribed treatment at index date 
group, and their interaction) and the selected factors. The associations with the 1-year treatment status were quantified in terms of odds ratios (ORs) with corresponding $95 \%$ confidence intervals (CIs). All analyses were conducted using SAS software, version 9.3 (SAS Institute, Cary, NC).

\section{Results}

A total of 47,171 women were eligible for inclusion in the analyses; patient selection is depicted in Online Resource Figure S1. Of these women, 7,834 (17\%) experienced vertebral fracture, 3,599 (8\%) hip fracture, 34,396 (73\%) non-hip/ non-vertebral fracture, and 1,342 (3\%) had fractures at multiple sites. The mean (SD) age at index fracture was 63 (10) years; 9,168 (19\%) patients had a prior recorded diagnosis of osteoporosis, and 9,522 (20\%) had received osteoporosis medication at some point during baseline, including 4,936 (11\%) who were receiving osteoporosis therapy at the time of fracture (currently treated; Table 1). Conversely, 33,870 (72\%) women had neither an osteoporosis diagnosis nor osteoporosis therapy during the baseline period. Women with hip fracture tended to be older and had a slightly higher mean Charlson comorbidity index score as compared with women in the other fracture groups (Table 1). A higher proportion of women with vertebral fracture had a diagnosis of osteoporosis or osteopenia than those in other fracture groups.

Figure 1 depicts the proportion of women who received osteoporosis therapy within 90 days and 1 year post-fracture, overall and according to prior (baseline period) osteoporosis therapy. A total of $8,445(18 \%)$ received medication within

Fig. 1 Percentage of women prescribed with osteoporosis medication within 90 days and within 1 year post-fracture. Prior treatment was that dispensed during the baseline period; current treatment overlapped the index date. $O P$ osteoporosis, Script prescription
90 days post-fracture; most of these women $(6,656$ [79 \%]) had also received osteoporosis therapy during the baseline period. A total of 10,826 (23\%) women received medication within 1 year post-fracture, of which $68 \%$ had received osteoporosis medication during baseline.

Table 2 describes demographic and baseline characteristics for women according to whether they received osteoporosis therapy within 90 days or 1 year post-fracture. Patients who received therapy post-fracture tended to be older than patients who did not, and they were more likely to have vertebral fracture, a prior osteoporosis diagnosis, and baseline osteoporosis therapy and to be adherent to baseline therapy; they were less likely to be obese.

Results of regression analyses supported these findings (Table 3). The adjusted odds ratio (OR) of receiving therapy within 1 year post-fracture for women with baseline osteoporosis therapy versus no baseline osteoporosis therapy were significantly greater for women with vertebral fracture (OR 12.7, $95 \%$ CI 11.2-14.5), hip fracture (OR 15.2, 12.5-18.7), nonhip/non-vertebral fracture (OR 34.4, 31.7-37.3), or multiple fractures (OR 18.1, 13.1-25.0). The odds of receiving medication prescribed for osteoporosis within 1 year post-fracture were significantly greater for women 60 years of age and older as compared with those between the ages of 50 and 59 (Table 3). Likewise, the odds of therapy post-fracture were significantly greater for women who had a baseline diagnosis of osteoporosis or osteopenia. As compared with those who had vertebral fracture, patients with hip, non-vertebral/non-hip, or multiple fractures had significantly lower odds of receiving therapy within 1 year post-fracture. Obese patients and those with arthritis, cardiovascular disease, or hypertension also had significantly lower odds of receiving therapy.

\section{- Script dispensed $\leq 90$ days post-fracture}

Script dispensed $\leq 1$ year post-fracture

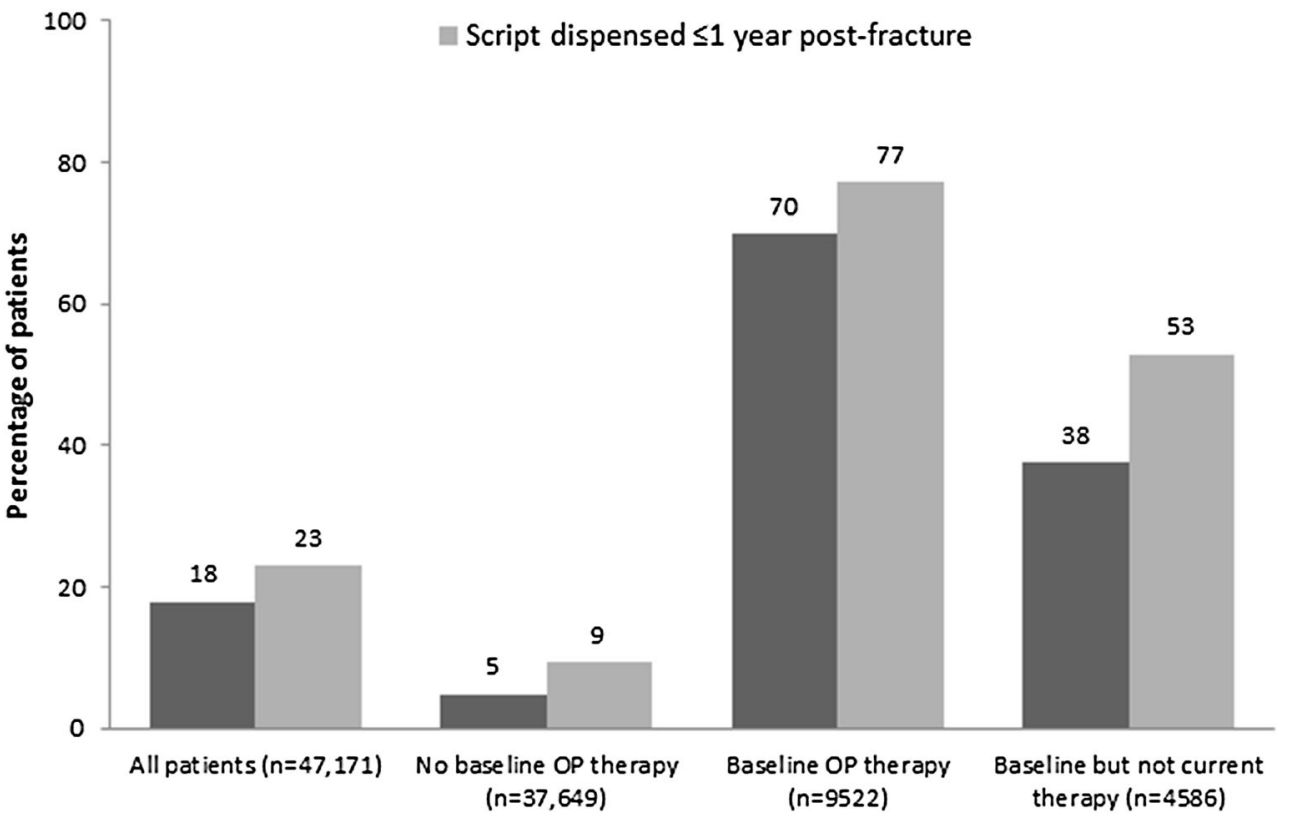


Table 2 Characteristics of women according to osteoporosis therapy dispensed during 1 year post-fracture

\begin{tabular}{|c|c|c|c|c|}
\hline \multirow[t]{2}{*}{ Characteristic } & \multicolumn{2}{|c|}{$\begin{array}{l}\text { Osteoporosis therapy dispensed } \\
\text { within } 90 \text { days post-fracture }\end{array}$} & \multicolumn{2}{|c|}{$\begin{array}{l}\text { Osteoporosis therapy dispensed } \\
\text { within } 1 \text { year post-fracture }\end{array}$} \\
\hline & Yes $(n=8,445)$ & No $(n=38,726)$ & Yes $(n=10,826)$ & No $(n=36,345)$ \\
\hline Age at index date, mean (SD) & $66.5(9.6)$ & $62.7(9.7)$ & $66.2(9.6)$ & $62.5(9.7)$ \\
\hline $50-59$ years & $2,481(29.4)$ & $18,450(47.6)$ & $3,338(30.8)$ & $17,593(48.4)$ \\
\hline $60-69$ years & $2,579(30.5)$ & $9,867(25.5)$ & $3,248(30.0)$ & $9,198(25.3)$ \\
\hline 70-79 years & $2,496(29.6)$ & $8,019(20.7)$ & $3,181(29.4)$ & $7,334(20.2)$ \\
\hline$\geq 80$ years & $889(10.5)$ & $2,390(6.2)$ & $1,059(9.8)$ & $2,220(6.1)$ \\
\hline \multicolumn{5}{|l|}{ Insurance type } \\
\hline HMO & $1,495(17.7)$ & $7,916(20.4)$ & $2,069(19.1)$ & $7,342(20.2)$ \\
\hline POS & $3,445(40.8)$ & $18,166(46.9)$ & $4,427(40.9)$ & $17,184(47.3)$ \\
\hline PPO & $943(11.2)$ & $3,349(8.6)$ & $1,170(10.8)$ & $3,122(8.6)$ \\
\hline Other & $2,562(30.3)$ & $9,295(24.0)$ & $3,160(29.2)$ & $8,697(23.9)$ \\
\hline Prior osteoporosis diagnosis & $4,212(49.9)$ & $4,956(12.8)$ & $4,921(45.5)$ & 4,247 (11.7) \\
\hline BMD assessment performed $^{\mathrm{a}}$ & $4,426(52.4)$ & $8,954(23.1)$ & $5,193(48.0)$ & $8,187(22.5)$ \\
\hline History of falls of any type resulting in medical services & $634(7.5)$ & $2,836(7.3)$ & $819(7.6)$ & $2,651(7.3)$ \\
\hline Baseline osteoporosis medication & $6,656(78.8)$ & $2,866(7.4)$ & $7,364(68.0)$ & $2,158(5.9)$ \\
\hline Pre-index MPR, mean (SD) & $0.57(0.30)$ & $0.26(0.22)$ & $0.54(0.30)$ & $0.24(0.22)$ \\
\hline Adherent patients ${ }^{\mathrm{b}}, n(\%$ of those on baseline $\mathrm{Rx})$ & $2,101(31.6)$ & $67(2.3)$ & $2,122(28.8)$ & $46(2.1)$ \\
\hline Currently treated ${ }^{\mathrm{c}}$ & $4,936(58.4)$ & $\mathrm{n} / \mathrm{a}$ & $4,936(45.6)$ & $\mathrm{n} / \mathrm{a}$ \\
\hline Not currently treated & $1,720(20.4)$ & $2,866(7.4)$ & $2,428(22.4)$ & $2,158(5.9)$ \\
\hline \multicolumn{5}{|l|}{ Index fracture group } \\
\hline Vertebral & $2,400(28.4)$ & $5,434(14.0)$ & $2,936(27.1)$ & $4,898(13.5)$ \\
\hline Hip & $663(7.9)$ & $2,936(7.6)$ & $898(8.3)$ & $2,701(7.4)$ \\
\hline Non-hip/non-vertebral & $5,095(60.3)$ & $29,301(75.7)$ & $6,604(61.0)$ & $27,792(76.5)$ \\
\hline Multiple & $287(3.4)$ & $1,055(2.7)$ & $388(3.6)$ & $954(2.6)$ \\
\hline CCI score, mean (SD) & $1.1(1.5)$ & $1.0(1.5)$ & $1.1(1.5)$ & $1.0(1.6)$ \\
\hline \multicolumn{5}{|l|}{ Key comorbid conditions } \\
\hline Osteopenia & $3,016(35.7)$ & $5,787(14.9)$ & $3,622(33.5)$ & $5,181(14.3)$ \\
\hline Arthritis & $4,867(57.6)$ & $20,125(52.0)$ & $6,218(57.4)$ & $18,774(51.7)$ \\
\hline Musculoskeletal pain & $4,120(48.8)$ & $16,133(41.7)$ & $5,248(48.5)$ & $15,005(41.3)$ \\
\hline Cardiovascular disease & $1,227(14.5)$ & $5,356(13.8)$ & $1,597(14.8)$ & $4,986(13.7)$ \\
\hline Myocardial infarction & $203(2.4)$ & $1,039(2.7)$ & $264(2.4)$ & $978(2.7)$ \\
\hline Hypertension & $4,733(56.0)$ & $20,881(53.9)$ & $6,060(56.0)$ & $19,554(53.8)$ \\
\hline Cerebrovascular disease & $1,082(12.8)$ & 4,031 (10.4) & $1,383(12.8)$ & $3,730(10.3)$ \\
\hline Chronic pulmonary disease & $1,949(23.1)$ & $8,169(21.1)$ & $2,537(23.4)$ & $7,581(20.9)$ \\
\hline Respiratory disease & $3,383(40.1)$ & $14,453(37.3)$ & $4,324(39.9)$ & $13,512(37.2)$ \\
\hline Hypothyroidism & $1,967(23.3)$ & $7,903(20.4)$ & $2,517(23.2)$ & $7,353(20.2)$ \\
\hline Hyperthyroidism & $201(2.4)$ & $818(2.1)$ & $271(2.5)$ & $748(2.1)$ \\
\hline Diabetes & $1,23514.6)$ & $7,118(18.4)$ & $1,624(15.0)$ & $6,729(18.5)$ \\
\hline Obesity & $354(4.2)$ & $3,109(8.0)$ & $467(4.3)$ & $2,996(8.2)$ \\
\hline Alzheimer's disease, dementia, depression, anxiety, sleep disorder & $2,551(30.2)$ & $12,233(31.6)$ & $3,360(31.0)$ & $11,424(31.4)$ \\
\hline Bulimia nervosa, anorexia nervosa, or anorexia & $588(7.0)$ & $2,082(5.4)$ & $739(6.8)$ & $1,931(5.3)$ \\
\hline Kidney disease & $561(6.6)$ & $2,380(6.1)$ & $699(6.5)$ & $2,242(6.2)$ \\
\hline Vitamin D deficiency & $309(3.7)$ & $1,036(2.7)$ & $378(3.5)$ & $967(2.7)$ \\
\hline \multicolumn{5}{|l|}{ Concomitant medication use } \\
\hline Gastroprotective agent & $2,541(30.1)$ & $8,968(23.2)$ & $3,194(29.5)$ & $8,315(22.9)$ \\
\hline NSAIDs & $3,552(42.1)$ & $13,832(35.7)$ & $4,534(41.9)$ & $12,850(35.4)$ \\
\hline Glucocorticoids & $2,725(32.3)$ & $10,171(26.3)$ & $3,451(31.9)$ & $9,445(26.0)$ \\
\hline
\end{tabular}


Table 2 (continued)

\begin{tabular}{|c|c|c|c|c|}
\hline \multirow[t]{2}{*}{ Characteristic } & \multicolumn{2}{|c|}{$\begin{array}{l}\text { Osteoporosis therapy dispensed } \\
\text { within } 90 \text { days post-fracture }\end{array}$} & \multicolumn{2}{|c|}{$\begin{array}{l}\text { Osteoporosis therapy dispensed } \\
\text { within } 1 \text { year post-fracture }\end{array}$} \\
\hline & Yes $(n=8,445)$ & No $(n=38,726)$ & Yes $(n=10,826)$ & No $(n=36,345)$ \\
\hline Estrogen & $1,538(18.2)$ & $6,941(17.9)$ & $1,996(18.4)$ & $6,483(17.8)$ \\
\hline
\end{tabular}

Data are $n$ (column \%) unless otherwise indicated

$B M D$ bone mineral density, $C C I$ Charlson comorbidity index, $H M O$ health maintenance organization, $M P R$ medication possession ratio, $n / a$ not applicable, NSAID non-steroidal anti-inflammatory drug, $P O S$ point of service, $P P O$ preferred provider organization, $R x$ therapy

${ }^{\mathrm{a}} \mathrm{BMD}$ results were not available from the database

${ }^{\mathrm{b}}$ Adherence defined as MPR $\geq 0.80$

${ }^{\mathrm{c}}$ Current treatment at index date

Table 3 Multivariable logistic regression on association between post-fracture treatment (within 1 year) and pre-fracture patient characteristics

\begin{tabular}{|c|c|c|c|}
\hline Variables & Adjusted Odds ratio & $95 \% \mathrm{CI}$ & $P$ value \\
\hline Index fracture site & & & $<0.001$ \\
\hline Hip fracture only vs. vertebral fracture only & 0.53 & $(0.47-0.60)$ & \\
\hline Non-hip/non-vertebral fracture only vs. vertebral fracture only & 0.29 & $(0.26-0.31)$ & \\
\hline Fractures at multiple sites vs. vertebral fracture only & 0.56 & $(0.46-0.68)$ & \\
\hline Treatment vs. non-treatment before the index fracture & & & $<0.001$ \\
\hline Among women with vertebral fracture only & 12.73 & $(11.17-14.50)$ & \\
\hline Among women with hip fracture only & 15.24 & $(12.45-18.66)$ & \\
\hline Among women with non-hip/non-vertebral fracture only & 34.35 & $(31.67-37.26)$ & \\
\hline Among women with fracture at multiple sites & 18.09 & $(13.10-24.99)$ & \\
\hline Age group & & & $<0.001$ \\
\hline $60-69$ vs. $50-59$ & 1.31 & $(1.22-1.42)$ & \\
\hline $70-79$ vs. $50-59$ & 1.68 & $(1.55-1.82)$ & \\
\hline 80 or above vs. $50-59$ & 1.37 & $(1.22-1.55)$ & \\
\hline \multicolumn{4}{|l|}{ Pre-fracture concomitant medication use } \\
\hline Gastroprotective agents & 1.05 & $(0.98-1.13)$ & 0.14 \\
\hline NSAIDs & 1.15 & $(1.08-1.22)$ & $<0.001$ \\
\hline Glucocorticoid & 1.16 & $(1.08-1.24)$ & $<0.001$ \\
\hline Estrogen & 1.08 & $(1.00-1.17)$ & 0.038 \\
\hline \multicolumn{4}{|l|}{ Pre-fracture diagnosis of comorbid conditions } \\
\hline Osteoporosis & 1.55 & $(1.44-1.66)$ & $<0.001$ \\
\hline Osteopenia & 1.41 & $(1.32-1.51)$ & $<0.001$ \\
\hline Arthritis & 0.93 & $(0.87-0.99)$ & 0.023 \\
\hline Musculoskeletal pain & 0.95 & $(0.89-1.01)$ & 0.099 \\
\hline Respiratory disease & 0.93 & $(0.87-0.99)$ & 0.024 \\
\hline Hyperthyroidism & 1.15 & $(0.95-1.40)$ & 0.14 \\
\hline Alzheimer's disease, dementia, depression, anxiety, sleep disorder & 0.96 & $(0.90-1.02)$ & 0.16 \\
\hline Cardiovascular disease & 0.89 & $(0.81-0.96)$ & 0.006 \\
\hline Kidney disease & 0.88 & $(0.78-1.00)$ & 0.044 \\
\hline Obesity & 0.70 & $(0.62-0.80)$ & $<0.001$ \\
\hline Hypertension & 0.89 & $(0.83-0.95)$ & $<0.001$ \\
\hline Vitamin D deficiency & 0.84 & $(0.71-1.00)$ & 0.052 \\
\hline Bulimia nervosa, anorexia nervosa, or anorexia & 0.80 & $(0.58-1.11)$ & 0.18 \\
\hline
\end{tabular}

CI confidence interval, NSAID non-steroidal anti-inflammatory drug 
Adherence to therapy during both baseline and postfracture was significantly higher for women who had a baseline diagnosis of osteoporosis than for those who did not (Table 4). Women with osteoporosis diagnosis represented slightly over half ( $57 \%$ ) of patients treated during baseline and slightly under half (46\%) of those treated post-fracture. Among the women who received therapy during the baseline period but not within 1 year post-fracture, adherence to baseline therapy was very low ( $2 \%$ of patients adherent during baseline) as compared with those who received both baseline and post-fracture therapy (29\% adherent during baseline) (Table 2).

Table 5 summarizes patient adherence to baseline and postfracture therapy according to fracture group and osteoporosis medication class. Approximately $75 \%$ of patients who were prescribed with osteoporosis therapy during either baseline or post-fracture received a bisphosphonate, and another $7 \%$ were switched between bisphosphonate and nonbisphosphonate therapy. There were several significant differences among the fracture groups in patient adherence to therapy during baseline and post-fracture. Women with hip fracture had the lowest adherence to bisphosphonates and overall to therapy during baseline and post-fracture, with only $21 \%$ of patients adherent to therapy post-fracture. Women with multiple fractures also showed poor adherence during the post-fracture year, with $23 \%$ adherent overall in contrast to 29 and $32 \%$ of patients with vertebral and non-hip/non-vertebral fractures, respectively (Table 5).

\section{Discussion}

This large retrospective study found that only $18 \%$ of the women included in the analysis received osteoporosis therapy within 90 days post-fracture, and less than a quarter overall
(23\%) received therapy within the first year post-fracture. These findings indicate that older women frequently do not receive pharmacologic therapy for osteoporosis after their first fracture. Lack of therapy was especially pronounced for women with non-hip/non-vertebral fracture, of whom only $19 \%$ received therapy within 1 year post-fracture, followed in frequency by those with hip fracture ( $25 \%)$. Patients who had a prior diagnosis of osteoporosis or osteopenia had significantly higher odds of receiving therapy post-fracture. Moreover, baseline medication use was a good predictor of postfracture medication use, as $77 \%$ of women who had received prior osteoporosis therapy during baseline subsequently received therapy at or after their first fracture.

Other studies have reported low rates of pharmacologic therapy after osteoporotic fracture. In Canada, Leslie et al. [15] found that therapy was initiated in the year after fracture for only $10 \%$ of patients in $2005 / 2006$, falling to $6 \%$ in $2007 / 2008$. The corresponding figure for our study was $9 \%$ of patients without prior baseline therapy who initiated osteoporosis therapy within 1 year after fracture. Moreover, because more than half of the $18 \%$ of women who initiated therapy within 90 days post-fracture were already receiving current therapy, essentially only $7-8 \%$ of women started therapy within 90 days post-fracture. This number increased to include only another $5 \%$ of women at 1 year post-fracture (namely, from 18 to $23 \%$ overall). In an earlier systematic review of 37 studies [20], post-fracture therapy rates exceeded $10 \%$ in only six studies. Other studies report low rates of post-fracture therapy even for patients with hip fracture and those hospitalized for fracture $[16,17,19]$. Our findings highlight the importance of assessing older women with non-hip/non-vertebral fractures as these women represented the majority of our study population $(73 \%)$ and had the lowest rates of osteoporosis baseline therapy, osteoporosis diagnosis, and post-fracture therapy of the four fracture groups.

Table 4 Baseline and post-fracture osteoporosis treatment status and adherence, overall and by baseline osteoporosis diagnosis

\begin{tabular}{|c|c|c|c|c|}
\hline & \multirow{2}{*}{$\begin{array}{l}\text { All women } \\
(n=47,171)\end{array}$} & \multicolumn{2}{|c|}{ Osteoporosis diagnosis at baseline } & \multirow[t]{2}{*}{$P$ value $^{\mathrm{a}}$} \\
\hline & & Yes & No & \\
\hline \multicolumn{5}{|l|}{ Baseline period osteoporosis therapy } \\
\hline Users, $n(\%)$ & $9,522(20.2)$ & $5,389(56.6)$ & $4,133(43.4)$ & \\
\hline Pre-index MPR, mean (SD) & $0.48(0.31)$ & $0.49(0.31)$ & $0.46(0.31)$ & $<0.001$ \\
\hline Adherent patients ${ }^{\mathrm{b}}, n$ (\% of users) & $2,168(22.8)$ & $1,269(23.5)$ & $899(21.8)$ & 0.038 \\
\hline \multicolumn{5}{|l|}{ Post-fracture osteoporosis therapy } \\
\hline Users, $n(\%)$ & $10,826(23.0)$ & $4,921(45.5)$ & $5,905(54.5)$ & \\
\hline Post-fracture MPR, mean (SD) & $0.55(0.31)$ & $0.60(0.30)$ & $0.51(0.31)$ & $<0.001$ \\
\hline Adherent patients ${ }^{\mathrm{b}}, n$ ( $\%$ of users) & $3,227(29.8)$ & $1,739(35.3)$ & $1,488(25.2)$ & $<0.001$ \\
\hline
\end{tabular}


Table 5 Osteoporosis therapy during the baseline period and within 1 year post-fracture: all women and by index fracture group

\begin{tabular}{|c|c|c|c|c|c|c|}
\hline \multirow[b]{2}{*}{ Baseline osteoporosis therapy by drug class } & \multirow{2}{*}{$\begin{array}{l}\text { All women } \\
(n=47,171)\end{array}$} & \multicolumn{4}{|l|}{ Fracture group } & \multirow[b]{2}{*}{$P$ value } \\
\hline & & $\begin{array}{l}\text { Vertebral } \\
(n=7,834)\end{array}$ & $\begin{array}{l}\text { Hip } \\
(n=3,599)\end{array}$ & $\begin{array}{l}\text { Non-hip/non-vertebral } \\
(n=34,396)\end{array}$ & $\begin{array}{l}\text { Multiple } \\
(n=1,342)\end{array}$ & \\
\hline \multicolumn{7}{|l|}{ Baseline osteoporosis treatment overall } \\
\hline Users, $n(\%)$ & $9,522(20.2)$ & $2,171(27.7)$ & $727(20.2)$ & $6,291(18.3)$ & $333(24.8)$ & \\
\hline Pre-index MPR, mean (SD) & $0.48(0.31)$ & $0.48(0.32)$ & $0.46(0.31)$ & $0.47(0.31)$ & $0.51(0.31)$ & 0.075 \\
\hline Adherent patients, $n$ ( $\%$ of users) & $2,168(22.8)$ & $532(24.5)$ & $145(19.9)$ & $1413(22.5)$ & $78(23.4)$ & 0.059 \\
\hline \multicolumn{7}{|l|}{ Bisphosphonate use only } \\
\hline Users, $n(\%)$ & $7,286(76.5)$ & $1,607(74.0)$ & $532(73.2)$ & $4,875(77.5)$ & $272(81.7)$ & \\
\hline Pre-index MPR, mean (SD) & $0.47(0.31)$ & $0.48(0.32)$ & $0.44(0.31)$ & $0.46(0.31)$ & $0.49(0.31)$ & 0.031 \\
\hline Adherent patients ${ }^{\mathrm{a}}, n$ ( $\%$ of users) & $1,567(21.5)$ & $377(23.5)$ & $91(17.1)$ & $1,040(21.3)$ & $59(21.7)$ & 0.020 \\
\hline \multicolumn{7}{|l|}{ Non-bisphosphonate use only } \\
\hline Users, $n(\%)$ & $1,538(16.2)$ & $361(16.6)$ & $136(18.7)$ & $1,002(15.9)$ & $39(11.7)$ & \\
\hline Pre-index MPR, mean (SD) & $0.47(0.32)$ & $0.42(0.33)$ & $0.47(0.33)$ & $0.48(0.31)$ & $0.53(0.32)$ & 0.008 \\
\hline Adherent patients, $n$ ( $\%$ of users) & $354(23.0)$ & $72(19.9)$ & $35(25.7)$ & $237(23.7)$ & $10(25.6)$ & 0.41 \\
\hline \multicolumn{7}{|l|}{ Bisphosphonate and non-bisphosphonate use ${ }^{b}$} \\
\hline Users, $n(\%)$ & $698(7.3)$ & $203(9.4)$ & $59(8.1)$ & $414(6.6)$ & $22(6.6)$ & \\
\hline Pre-index MPR, mean (SD) & $0.61(0.29)$ & $0.62(0.30)$ & $0.56(0.29)$ & $0.60(0.28)$ & $0.66(0.27)$ & 0.47 \\
\hline Adherent patients, $n$ ( $\%$ of users) & $247(35.4)$ & $83(40.9)$ & $19(32.2)$ & $136(32.9)$ & $9(40.9)$ & 0.22 \\
\hline Post-fracture osteoporosis therapy by drug class & $\begin{array}{l}\text { All patients } \\
\qquad(n=47,171)\end{array}$ & $\begin{array}{l}\text { Vertebral } \\
\qquad(n=7,834)\end{array}$ & $\begin{array}{l}\text { Hip } \\
\qquad(n=3,599)\end{array}$ & $\begin{array}{l}\text { Non-hip/non-vertebral } \\
\quad(n=34,396)\end{array}$ & $\begin{array}{l}\text { Multiple } \\
\qquad(n=1,342)\end{array}$ & $P$ value \\
\hline \multicolumn{7}{|l|}{ Post-fracture osteoporosis treatment overall } \\
\hline Users, $n(\%)$ & $10,826(23.0)$ & $2,936(37.5)$ & $898(25.0)$ & $6,604(19.2)$ & $388(28.9)$ & \\
\hline Post-fracture MPR, mean (SD) & $0.55(0.31)$ & $0.55(0.31)$ & $0.51(0.30)$ & $0.56(0.31)$ & $0.51(0.29)$ & $<0.001$ \\
\hline Adherent patients, $n$ ( $\%$ of users) & $3,227(29.8)$ & $864(29.4)$ & $190(21.2)$ & $2083(31.5)$ & $90(23.2)$ & $<0.001$ \\
\hline \multicolumn{7}{|l|}{ Bisphosphonate use only } \\
\hline Users, $n(\%)$ & $8,243(76.1)$ & $1,979(67.4)$ & $692(77.1)$ & $5,276(79.9)$ & $296(76.3)$ & \\
\hline Post-fracture MPR, mean (SD) & $0.54(0.30)$ & $0.55(0.31)$ & $0.49(0.29)$ & $0.55(0.31)$ & $0.51(0.29)$ & $<0.001$ \\
\hline Adherent patients ${ }^{\mathrm{a}}, n$ ( $\%$ of users) & $2,364(28.7)$ & $577(29.2)$ & $131(18.9)$ & $1,592(30.2)$ & 64 (21.6) & $<0.001$ \\
\hline \multicolumn{7}{|l|}{ Non-bisphosphonate use only } \\
\hline Users, $n(\%)$ & $1,782(16.5)$ & $594(20.2)$ & $141(15.7)$ & $989(15.0)$ & $58(14.9)$ & \\
\hline Post-fracture MPR, mean (SD) & $0.53(0.32)$ & $0.45(0.32)$ & $0.47(0.30)$ & $0.57(0.31)$ & $0.46(0.32)$ & $<0.001$ \\
\hline Adherent patients, $n$ ( $\%$ of users) & $501(28.1)$ & $126(21.2)$ & $29(20.6)$ & $333(33.7)$ & $13(22.4)$ & $<0.001$ \\
\hline \multicolumn{7}{|l|}{ Bisphosphonate and non-bisphosphonate use ${ }^{b}$} \\
\hline Users, $n(\%)$ & $801(7.4)$ & $363(12.4)$ & $65(7.2)$ & $339(5.1)$ & $34(8.8)$ & \\
\hline Post-fracture MPR, mean (SD) & $0.69(0.26)$ & $0.69(0.26)$ & $0.69(0.26)$ & $0.70(0.26)$ & $0.62(0.26)$ & 0.45 \\
\hline Adherent patients, $n$ ( $\%$ of users) & $362(45.2)$ & $161(44.4)$ & $30(20.6)$ & $158(46.6)$ & $13(22.4)$ & 0.79 \\
\hline
\end{tabular}

$M P R$ medication possession ratio

${ }^{a}$ Adherence defined as MPR $\geq 0.80$

${ }^{\mathrm{b}}$ Patients were switched between bisphosphonate and non-bisphosphonate (not concomitant use)

Several reasons have been proposed for the under-prescribing of osteoporosis therapies after fracture. In some settings, there is lack of coordination of care: For example, orthopedic surgeons may not initiate medical therapy or communicate with primary care physicians regarding their patients with osteoporotic fractures [28]. Another potential reason is patient or physician concerns about the tolerability of therapy; limited data suggest patient concerns [29] and that some physicians may not prescribe treatment because of concerns about adverse effects [28]. Reported strategies shown to improve the post-fracture treatment gap include multidisciplinary interventions to educate and motivate both patients and their health care providers [30-32]. Work by Beaton et al. [33] has identified the importance of patientperceived need for therapy in predicting the initiation of therapy 
after fracture. Further education regarding osteoporosis management including fracture liaison services programs or adherence programs may lead to improved outcomes and lower health care resource use and costs [34-36].

A strength of this study is the large patient population and the fact that we followed women for 3 consecutive years including 1 year post-fracture. Moreover, the results depict current treatment practices in the USA, drawn from real-life patient data recorded from 2003 to mid-2012. We did not investigate the reasons for under-treatment in the present study but instead sought to characterize women who did not receive osteoporosis therapy post-fracture.

A limitation of our analyses, as for all studies using administrative claims data, is that inaccuracies associated with coding errors could lead to misclassification of patients. Moreover, the analyses relied on prescription claims as a proxy for medication use. While this method gives a clear indication of dispensing patterns, the data on adherence are less reliable because we had no way to track whether women actually took the medication as prescribed. Moreover, the dataset does not include a record of prescriptions that were not filled; therefore, our findings could represent a combination of under-prescribing by health care providers as well as patient non-compliance with initiating prescribed therapy. It is not possible from the database to identify with certainty the specialty of the treating physicians. In addition, it is possible that falls were under-reported and that we included some fractures that were not osteoporotic, although we restricted the patient population to women 50 years of age and older and excluded fractures that are not considered osteoporotic by expert consensus [37]. Finally, we cannot rule out unrecognized confounding factors subjecting our results to bias.

The post-fracture treatment gap is a well-recognized problem for patients with osteoporotic fracture, exposing them to increased risk of subsequent fracture. The results of this study indicate that lack of post-fracture pharmacotherapy remains a problem among older women with osteoporotic fracture in the US and constitutes an important unmet need for preventing subsequent fracture. Our findings highlight the need for physician education about osteoporosis risk assessment and for increased patient education and involvement in their care to help reduce the risk and occurrence of osteoporotic fractures and overall health care burden as a result.

Acknowledgments This study was funded by Merck Sharp \& Dohme Corp., Whitehouse Station, NJ, USA. Medical writing assistance was provided by Elizabeth V. Hillyer, DVM. This assistance was funded by Merck Sharp \& Dohme Corp., a subsidiary of Merck \& Co., Inc., Whitehouse Station, NJ.

Ethical standards As this was a non-interventional study using anonymized patient data, ethical committee approval was not necessary.

Conflicts of interest AW was a fellow at Merck during the study. SS, $\mathrm{AM}$, and PM are employees of Merck and may own stock or stock options. C-PSF is an employee of Asclepius JT LLC, which served as consultant to Merck.

Open Access This article is distributed under the terms of the Creative Commons Attribution Noncommercial License which permits any noncommercial use, distribution, and reproduction in any medium, provided the original author(s) and the source are credited.

\section{References}

1. Cooper C, Cole ZA, Holroyd CR, Earl SC, Harvey NC, Dennison EM, Melton LJ, Cummings SR, Kanis JA (2011) Secular trends in the incidence of hip and other osteoporotic fractures. Osteoporos Int 22:1277-1288

2. Burge R, Dawson-Hughes B, Solomon DH, Wong JB, King A, Tosteson A (2007) Incidence and economic burden of osteoporosisrelated fractures in the United States, 2005-2025. J Bone Miner Res 22:465-475

3. Nguyen ND, Ahlborg HG, Center JR, Eisman JA, Nguyen TV (2007) Residual lifetime risk of fractures in women and men. J Bone Miner Res 22:781-788

4. Looker AC, Melton LJ 3rd, Borrud LG, Shepherd JA (2012) Lumbar spine bone mineral density in US adults: demographic patterns and relationship with femur neck skeletal status. Osteoporos Int 23:13511360

5. National Osteoporosis Foundation (2013) Clinician's guide to the prevention and treatment of osteoporosis. Washington DC. Available at http://nof.org/hcp/resources/913. Accessed 4 Feb 2014

6. Barrett-Connor E, Weiss TW, McHorney CA, Miller PD, Siris ES (2009) Predictors of falls among postmenopausal women: results from the National Osteoporosis Risk Assessment (NORA). Osteoporos Int 20:715-722

7. Leslie WD, Morin S, Lix LM, Johansson H, Oden A, McCloskey E, Kanis JA (2012) Fracture risk assessment without bone density measurement in routine clinical practice. Osteoporos Int 23:75-85

8. Sambrook PN, Flahive J, Hooven FH, Boonen S, Chapurlat R, Lindsay R, Nguyen TV, Diez-Perez A, Pfeilschifter J, Greenspan SL, Hosmer D, Netelenbos JC, Adachi JD, Watts NB, Cooper C, Roux C, Rossini M, Siris ES, Silverman S, Saag KG, Compston JE, LaCroix A, Gehlbach S (2011) Predicting fractures in an international cohort using risk factor algorithms without BMD. J Bone Miner Res 26:2770-2777

9. Siris E, Delmas PD (2008) Assessment of 10-year absolute fracture risk: a new paradigm with worldwide application. Osteoporos Int 19: 383-384

10. Kanis JA, Hans D, Cooper C, Baim S, Bilezikian JP, Binkley N, Cauley JA, Compston JE, Dawson-Hughes B, El-Hajj Fuleihan G, Johansson H, Leslie WD, Lewiecki EM, Luckey M, Oden A, Papapoulos SE, Poiana C, Rizzoli R, Wahl DA, McCloskey EV (2011) Interpretation and use of FRAX in clinical practice. Osteoporos Int 22:2395-2411

11. Kanis JA, McCloskey EV, Johansson H, Cooper C, Rizzoli R, Reginster JY (2013) European guidance for the diagnosis and management of osteoporosis in postmenopausal women. Osteoporos Int 24:23-57

12. Hopkins RB, Goeree R, Pullenayegum E, Adachi JD, Papaioannou A, Xie F, Thabane L (2011) The relative efficacy of nine osteoporosis medications for reducing the rate of fractures in post-menopausal women. BMC Musculoskelet Disord 12:209

13. MacLean C, Newberry S, Maglione M, McMahon M, Ranganath V, Suttorp M, Mojica W, Timmer M, Alexander A, McNamara M, Desai SB, Zhou A, Chen S, Carter J, Tringale C, Valentine D, Johnsen B, 
Grossman J (2008) Systematic review: comparative effectiveness of treatments to prevent fractures in men and women with low bone density or osteoporosis. Ann Intern Med 148:197-213

14. Shepherd AJ, Cass AR, Ray LA, Tan A, Wilkinson GS (2012) Treatment for older men with fractures. Osteoporos Int 23:1041-1051

15. Leslie WD, Giangregorio LM, Yogendran M, Azimaee M, Morin S, Metge C, Caetano P, Lix LM (2012) A population-based analysis of the post-fracture care gap 1996-2008: the situation is not improving. Osteoporos Int 23:1623-1629

16. Luthje P, Nurmi-Luthje I, Kaukonen JP, Kuurne S, Naboulsi H, Kataja M (2009) Undertreatment of osteoporosis following hip fracture in the elderly. Arch Gerontol Geriatr 49:153-157

17. Panneman MJ, Lips P, Sen SS, Herings RM (2004) Undertreatment with anti-osteoporotic drugs after hospitalization for fracture. Osteoporos Int 15:120-124

18. Dreinhofer KE, Anderson M, Feron JM, Herrera A, Hube R, Johnell O, Lidgren L, Miles K, Tarantino U, Simpson H, Wallace WA (2005) Multinational survey of osteoporotic fracture management. Osteoporos Int 16(Suppl 2):S44-S53

19. Shibli-Rahhal A, Vaughan-Sarrazin MS, Richardson K, Cram P (2011) Testing and treatment for osteoporosis following hip fracture in an integrated U.S. healthcare delivery system. Osteoporos Int 22: 2973-2980

20. Elliot-Gibson V, Bogoch ER, Jamal SA, Beaton DE (2004) Practice patterns in the diagnosis and treatment of osteoporosis after a fragility fracture: a systematic review. Osteoporos Int 15:767-778

21. Li L, Roddam A, Gitlin M, Taylor A, Shepherd S, Shearer A, Jick S (2012) Persistence with osteoporosis medications among postmenopausal women in the UK General Practice Research Database. Menopause 19:33-40

22. Hadji P, Claus V, Ziller V, Intorcia M, Kostev K, Steinle T (2012) GRAND: the German retrospective cohort analysis on compliance and persistence and the associated risk of fractures in osteoporotic women treated with oral bisphosphonates. Osteoporos Int 23:223-231

23. Optum Clinformatics ${ }^{\mathrm{TM}}$ Data Mart (2012) OptumInsight. Available at http://www.optuminsight.com/life-sciences/solutions/valuestrategy/marketing-analytics/clinformatics-data-mart/overview. Accessed 4 Feb 2014

24. National Center for Health Statistics International Classification of Diseases, Ninth Revision, Clinical Modification (ICD-9-CM). Available at http://www.cdc.gov/nchs/icd/icd $9 \mathrm{~cm} . h t m$. Accessed 4 Feb 2014

25. U.S. Food and Drug Administration National Drug Code Directory. Available at http://www.fda.gov/Drugs/InformationOnDrugs/ ucm142438.htm. Accessed 4 Feb 2014

26. Martin KE, Yu J, Campbell HE, Abarca J, White TJ (2011) Analysis of the comparative effectiveness of 3 oral bisphosphonates in a large managed care organization: adherence, fracture rates, and all-cause cost. J Manag Care Pharm 17:596-609

27. Deyo RA, Cherkin DC, Ciol MA (1992) Adapting a clinical comorbidity index for use with ICD-9-CM administrative databases. J Clin Epidemiol 45:613-619

28. Skedros JG, Holyoak JD, Pitts TC (2006) Knowledge and opinions of orthopaedic surgeons concerning medical evaluation and treatment of patients with osteoporotic fracture. J Bone Joint Surg Am 88:18 24

29. Sale JE, Gignac MA, Hawker G, Frankel L, Beaton D, Bogoch E, Elliot-Gibson V (2011) Decision to take osteoporosis medication in patients who have had a fracture and are 'high' risk for future fracture: a qualitative study. BMC Musculoskelet Disord 12:92

30. Roux S, Beaulieu M, Beaulieu MC, Cabana F, Boire G (2013) Priming primary care physicians to treat osteoporosis after a fragility fracture: an integrated multidisciplinary approach. J Rheumatol 40: 703-711

31. Chandran M, Tan MZ, Cheen M, Tan SB, Leong M, Lau TC (2013) Secondary prevention of osteoporotic fractures-an "OPTIMAL" model of care from Singapore. Osteoporos Int 24:2809-2817

32. McGowan BM, Bennett K, Marry J, Walsh JB, Casey MC (2011) Primary-care prescribing of anti-osteoporotic-type medications following hospitalisation for fractures. Eur J Clin Pharmacol 67:301308

33. Beaton DE, Dyer S, Jiang D, Sujic R, Slater M, Sale JE, Bogoch ER (2014) Factors influencing the pharmacological management of osteoporosis after fragility fracture: results from the Ontario Osteoporosis Strategy's fracture clinic screening program. Osteoporos Int 25:289296

34. Eisman JA, Bogoch ER, Dell R, Harrington JT, McKinney RE Jr, McLellan A, Mitchell PJ, Silverman S, Singleton R, Siris E (2012) Making the first fracture the last fracture: ASBMR task force report on secondary fracture prevention. J Bone Miner Res 27:2039-2046

35. Eekman DA, van Helden SH, Huisman AM, Verhaar HJ, Bultink IE, Geusens PP, Lips P, Lems WF (2014) Optimizing fracture prevention: the fracture liaison service, an observational study. Osteoporos Int 25:701-709

36. Solomon DH, Patrick AR, Schousboe J, Losina E (2014) The potential economic benefits of improved post-fracture care: a costeffectiveness analysis of a fracture liaison service in the US health care system. J Bone Miner Res. doi:10.1002/jbmr.2180

37. Diez-Perez A, Adachi JD, Agnusdei D, Bilezikian JP, Compston JE, Cummings SR, Eastell R, Eriksen EF, Gonzalez-Macias J, Liberman UA, Wahl DA, Seeman E, Kanis JA, Cooper C (2012) Treatment failure in osteoporosis. Osteoporos Int 23:2769-2774 